\title{
Value of serum sTREM-1,D-lactate and I-FABP in diagnosis and prognosis evaluation in liver cirrhotic patients with sepsis
}

\section{Danhong Yang ( $\nabla$ ydh-11@163.com )}

Zhejiang Provincal People's Hospital https://orcid.org/0000-0002-1235-942X

\section{Qiang Zhang}

Yijishan Hospital of Wannan Medical College

\section{Weiping Wang}

Bengbu Medical College

\section{Yicheng Huang}

Zhejiang Provincial People's Hospital

\section{Hongying Pan}

Zhejiang Provincial People's Hospital

\section{Miaomiao Wu}

Bengbu Medical College

Research article

Keywords: Liver cirrhosis,Sepsis,sTREM-1,D-lactate ,I-FABP

Posted Date: October 16th, 2020

DOI: https://doi.org/10.21203/rs.3.rs-90875/v1

License: (c) (i) This work is licensed under a Creative Commons Attribution 4.0 International License. Read Full License 


\section{Abstract}

\section{Background}

Sepsis is can cause serious negative effects among patients with cirrhosis.Finding reliable biomarker in early diagnose and prognosis in septic patients with liver cirrhosis is very important. We assessed value of serumsoluble triggering receptor expressed on myeloid cells-1 ( sTREM-1),D-lactate and Intestinal fatty acid binding protein(I-FABP) in diagnosis and prognosis evaluation in liver cirrhotic patients with sepsis.

\section{Methods}

A prospective observational study of 158 cirrhosis patients was conducted. A total of 158cirrhosis patients were enrolled, 83 of whom were diagnosed with sepsis and 75 patients without sepsis during hospitalization. We evaluated value of early diagnostic and prognosis of cells-1(sTREM-1),D-lactate and IFABP in cirrhotic patients with sepsis. Receiver operating characteristic curves (ROCs) were used to assess the ability of tested biomarkers to diagnose and prognosis cirrhotic patients with sepsis.

\section{Results}

Our results showed the CRP $\triangle \mathrm{PCT} \llbracket$ sTREM-1, D-lactate and I-FABP were significantly higher in the sepsis group than in the no-sepsis group.,but only levels of serum STREM-1 showed increasing trend with the severity of the cirrhotic patients with sepsis. The cutoff value of sTREM-1 was $123.62 \mathrm{pg} / \mathrm{mL}$ and showed $84.3 \%$ sensitivity and $92.0 \%$ specificity and high accuracy in the early diagnosis for cirrhotic patients with sepsis.

The AUC of sTREM- 1 was $0.959(95 \% \mathrm{Cl}$ : $0.934-0.985)$ and higher than that of CRP,PCT,D-lactate and I-FABP( $P<0.001, P=0.002, P<0.001, P<0.001$ respectively). Total 31 (37.35\%) cirrhotic patients with sepsis cases died during 90-day follow-up.Only sTREM-1 level had significance between survivors and nonsurvivors(263.99 \pm 213.79 vs $672.24 \pm 191.44, P=0.031)$. The AUCs of sTREM- 1 for predicting 90 -day mortality was 0.904 ( $95 \% \mathrm{Cl}: 0.836-.971)$ and higher than that of CRP,PCT,D-lactate $(P=0.001, p=0.003, P<0.001$ respectively)

The AUCs of I-FABP is only 0.482 and had no value of predicting prognosis.

\section{Conclusion}

Serum sTREM- 1 is a valuable new biomarker for early diagnosis and prognosis in cirrhosis with sepsis, proving more accurate than CRP,PCT, D - lactate and I - FABP.Serum D-lactate and I-FABP levels can identify early cirrhosis with sepsis, but they is little relationship with the prognosis of patients.

\section{Background}

Patients with liver cirrhosis have portal hypertension,increased intestinal permeability, the imbalance of intestinal flora and the decreased immunity, which altered defense against bacteri. This reduced bacterial clearance in cirrhotic patients can contribute to a high risk of systemic infection and progress easily to sepsis [1].The latter is a common and serious burden among cirrhotic patients because it can cause liver function to 
deteriorate further, and has serious negative effects on the disease course[2, 3]. Some evidence suggests that systemic inflammation in cirrhotic patients maximizes the risk of complications (e.g. portal hypertensive bleeding, hepatic encephalopathy, acute-on-chronic liver failure), acute renal insults [4],which may easily cause multiple organ dysfunction syndrome (MODS), leading to high mortality.About $30 \%$ of cirrhotic patients die within 1 month of infection and another $30 \%$ die within 1 year. We have reported that 90 -day mortality in cirrhotic patients with systemic inflammatory response syndrome(SIRS)can be up to $38 \%$ [5].Early diagnosis,recognition of severity and prognostic evaluation are helpful to improve prognosis and reduce mortality for cirrhotic patients with sepsis.At present, the diagnosis of sepsis mainly depends on nonspecific physiological conditions and culture based on pathogen detection, which leads to the uncertainty of diagnosis and the delay of treatment. The diagnostic value of traditional detection indexes including $\mathrm{C}$ reactive protein (CRP $\square$ and procalcitonin(PCT) is controversial.Silvestre JP et al [6] found CRP levels were significantly decreased in patients with sepsis and fulminant hepatic failure.Therefore, finding reliable biomarker in early diagnose and prognosis in septic patients with liver cirrhosis is very important.

Triggering receptor expressed on myeloid cells-1 (TREM-1) is a member of the immunoglobulin superfamilyexpressedon blood neutrophils andmature monocytes/macrophages [7].TREM-1 is a cell-surface receptor, playing a key-role in the septic process[6, 7]. In vitroand invivostudies have shown that TREM-1 expression is upregulated strongly by extracellular bacteria (particularly their cell-wall components) and fungi, although not by mycobacteria [8, 9]. sTREM-1 is a soluble form of TREM and is released from activated phagocytes [10].Many studied have indicated that sTREM-1 could be a reliable parameter in differentiating patients with sepsis from those with SIRS [11, 12]. Hence, sTREM-1 has attracted attention as a diagnostic/prognostic biomarker for various infectious diseases and sepsis[13, 14]. However, value of STREM-1 for the early diagnosis and prognosis evaluation in liver cirrhotic patients with sepsis has not been done.

In liver cirrhosis, disruption of the intestinal mucosal barrier function and increased mucosal intestinal permeability lead to bacterial translocation and endotoxemia, which increase incidence of systemic inflammatory response syndrome(SIRS) and infection $[15,16]$.Intestinal injury can cause sepsis to develop into septic shock,even multiple organ dysfunction( MDRO) [16]. Intestional tract is often the earliest site of sepsis complicated with organ dysfunction .With the increase of intestinal permeability, bacteria and their metabolites can enter the blood circulation including $D$-lactate which could evaluate intestinal injury and endoxemia following severe injuries[17, 18] .I-FABP is small-cytosolic watersoluble proteins in mature enterocytes. The level of I-FABP can reflected the physiological turnover rate of enterocyte, with elevated levels indicating intestinal epithelial cell damage[19]. The level of D-lactate and I-FABP were considered to be a useful marker for the early detection of significant intestinal injury [20,21].Nevertheless,little is known about the value of D-lactate and I-FABP in the early diagnosis and prognosis of cirrhosis with sepsis.

In the present study, we aimed to assess (1) performance of serum sTREM-1, D-lactate and I-FABP levels in the early diagnosis and prognosis ofcirrhotic patients with sepsis in comparison with routinely used traditional parameters such as CRP and PCT;(2)whether serum sTREM-1, D-lactate and I-FABP levels are able to identify severity and provide progress information during sepsis episodes in cirrhosis . 


\section{Materials And Methods}

\section{Definitions and criteria}

A total of 158 cirrhotic patients with sepsis admitted to and treated at our hospital from September 2016 to December 2019 were analyzed. The study protocol was approved by the Bioethics Committee of Zhejiang Provincial People's Hospital. Written informed consent was obtained from individual or guardian participants.Sepsis were diagnosed with sepsis, severe sepsis or septic shock, according to the 2015 Definition . Inclusion criteria: a) age $\geq 18$ years; b) Diagnosis of cirrhosis established by either histology,or clinical data .c)sepsis due one of the following infections: Septicemia,pneumonia , acute pyelonephritis, intrabdominal infection, primary bacteremia, ventilator-associated pneumonia (VAP) , acute episode of chronic ,cholecystitis ,cholangitis,pleural infection,catheter related infection,urinary tract infection,and so on.Demographic, clinical, laboratory, imaging and pathological data were collected during the patient's hospital stay.

The study protocol was in agreement with the Declaration of Helsinki.Patients with cirrhosis were divided into Sepsis group (83 cases) and no-sepsis group (75 cases). Sepsis patients were divided into general sepsis group (33 cases), severe sepsis group (28 cases), septic shock group (22 cases) according to the severity of the disease, and survival group (54 cases) and death group (29 cases) according to the 90 days outcome.The association of patients with or without sepsis was assessed by microbiologic cultures. Patients with bacterial infection were treated with antibiotics.

Exclusion criteria were: age < 18 years ; HIV infection; pregnancy; treatment forimmunosuppression; severe cardiopulmonary disorders; patients on steroids or other immunosuppressive therapy;patients with certain autoimmune disorders and collagen disease such as hyperthyroidism,systemic lupus erythematosus (SLE), dermatomyositis, scleroderma, and so on;Patients were evaluated within $12 \mathrm{~h}$ for sepsis according to the criteria $[22,23]$. The final diagnosis was based on other clinical data, including diagnostic imaging and microbiological results.

\section{Follow-up}

Data of discharged patients were obtained by telephone contact to patients or their family. All patients in the cohort were studied for 90 days or until death.

\section{Laboratory examination}

Routine microbiology examination for sepsis patients involved more than one pair of blood cultures. Analyses of urine, sputum, bronchoalveolar lavage fluid, cerebrospinal fluid, abscesses and closed wounds were undertaken. Blood was drawn immediately after presentation to the Emergency Department of Zhejiang Provincial People's Hospital and analyzed in the laboratory within $12 \mathrm{~h}$ for measurement of WBC counts, neutrophil percentage, $\mathrm{C}$-reactive protein(CRP) , procalcitonin(PCT) , and blood chemistry.A particle-enhanced immunoturbidimetric methodforquantitative determination of whole-range CRP was used employing a kit from Shanghai Upper Biotech Pharma (Shanghai, China). Procalcitonin levels were measured using a Cobas E601 chemiluminescence analyzer (8000 series; Roche, Basel, Switzerland).Additional serum samples were 
collected, aliquots were prepared, and samples frozen at $-80^{\circ} \mathrm{C}$ until analyses of sTREM- 1 . sTREM- 1 levels were determined in duplicate using a double-antibody sandwich enzyme-linked immunosorbent assay (ELISA) (Quantikine ${ }^{\circledR}$ Human TREM-1).D-lactate levels were measured by abcam,ab83429,D-lactate colorimetric Assay Kit.Serum I-FABP concentration was assayed by ELIAS ( Human FABP2 ELISA Kit,abcam).

\section{Statistical analyses}

Descriptive data are the mean \pm standard deviation or number (percentage). Comparison of continuous variables was carried out using the Student's $t$-test. Skewness distribution data are the median with range (25-75 interquartile range) and were analyzed using the Mann-Whitney U-test. Categorical variables were analyzed using Fisher's exact test or Pearson's $\chi^{2}$-test. Evaluation of the early diagnostic performance and 90-days mortality of CRP,PCT,sTREM-1 , D-lactate and I-FABP was done using receiver operating characteristic curves (ROCs). The latter were compared using a non-parametric method. The cutoff value, which was the maximum area under the ROC curve (AUC), and accuracies were calculated with 95\% confidence intervals.) AUCs of the different indices were compared. $P<0.05$ was considered significant. SPSS 22.0 (IBM, Armonk, NY, USA) was used for all analyses.

\section{Results}

\section{Patients' characteristics}

Detailed characterization of the 158 patients is shown in Table 1. More patients had a "mixed" etiology (alcohol + HBV) in the cirrhotic patients in sepsis group compared with the cirrhotic patients in no- sepsis group (22.89[19/83] vs 5.08\% [6/75]) $(P=0.015)$. There was no significant difference between sepsis group and no-sepsis group in terms of age $(P=0.223)$, sex ratio $(P=0.906)$, alanine transaminase level $(P=0.899)$ or the proportion of people with Child-Pugh class-B disease $(P=0.872)$. Cirrhotic patients in sepsis group had significantly higher total bilirubin $(P<0.001)$, prothrombin time $(P<0.001)$, creatinine $(P<0.001)$, proportion of Child-Pugh class-C $(P<0.001)$, Child-Pugh score $(P<0.00)$, as well as a lower albumin level $(P<0.001)$, platelet count $(P<0.001)$, and proportion of Child-Pugh class-A disease $(P<0.001)$, compared with patients without sepsis group. Cirrhotic patients in sepsis group has more higher 90 - day mortality $(P<$ 0.001). (Table 1).

Table 1. Comparative clinical features of cirrhotic patients with and without sepsis during hospitalization ( $\mathrm{n}=$ 158) 


\begin{tabular}{|c|c|c|c|c|}
\hline Characteristics & $\begin{array}{l}\text { Total } \\
(n=158)\end{array}$ & $\begin{array}{l}\text { Sepsis group } \\
(n=83)\end{array}$ & $\begin{array}{l}\text { No-sepsisgroup } \\
(n=75)\end{array}$ & Pvalue \\
\hline Age (yrs) & $62.37 \pm 13.64$ & $65.04 \pm 13.67$ & $59.23 \pm 11.53$ & 0.223 \\
\hline Gender (male\%) & $63.29(100 / 158)$ & $63.85(53 / 83)$ & $62.67(47 / 75)$ & 0.906 \\
\hline \multicolumn{5}{|l|}{ Etiologies of cirrhosis } \\
\hline Viral hepatitis & $43.67(69 / 158)$ & $39.76(33 / 83)$ & $0.48(36 / 75)$ & 0.201 \\
\hline HBV & $41.77(66 / 158)$ & $37.35(31 / 83)$ & $46.67(35 / 75)$ & 0.261 \\
\hline $\mathrm{HCV}$ & $1.90(3 / 158)$ & $2.41(2 / 83)$ & $1.33(1 / 75)$ & 1.000 \\
\hline Alcohol & $13.29(21 / 158)$ & $10.75(9 / 83)$ & $16.00(12 / 75)$ & 0.359 \\
\hline Mixed etiology & $18.99(30 / 158)$ & $27.71(23 / 83)$ & $9.33(7 / 75)$ & 0.004 \\
\hline $\mathrm{HBV}+\mathrm{HEV}$ & $1.90(3 / 158)$ & $2.41(2 / 83)$ & $1.33(1 / 75)$ & 1.000 \\
\hline HBV + alcohol & $15.82(25 / 158)$ & $22.89(19 / 83)$ & $8.00(6 / 75)$ & 0.015 \\
\hline $\begin{array}{c}\text { Alcoholt } \\
\text { Schistosomal }\end{array}$ & $1.27(2 / 158)$ & $2.41(2 / 83)$ & 0 & 0.498 \\
\hline Schistosomal cirrhos is & $3.16(5 / 158)$ & $2.41(2 / 83)$ & $4.00(3 / 75)$ & 0.669 \\
\hline Autoimmune hepatitis & $6.96(11 / 158)$ & $7.22(6 / 83)$ & $6.67(5 / 75)$ & 0.774 \\
\hline Cryptogenic cirrhosis & $13.92(22 / 158)$ & $12.05(10 / 83)$ & $16.00(12 / 75)$ & 0.657 \\
\hline $\operatorname{ALT}(\mathrm{U} / \mathrm{L}, \quad \bar{x} \pm 5)$ & $35.11(21,38)$ & $38.66(20.25,40.25)$ & $\begin{array}{l}31.27(22.00 \\
38.00)\end{array}$ & 0.899 \\
\hline $\mathrm{ALB}(g / \mathrm{L}, \bar{x} \pm 5)$ & $29.83 \pm 4.14$ & $27.98 \pm 4.38$ & $31.82 \pm 2.71$ & $<0.001$ \\
\hline $\mathrm{TB}(\mathrm{mmol} / \mathrm{L}, \bar{x} \pm 5)$ & $49.37(25.8,51.00)$ & $65.89(32.00,76.13)$ & $31.45 \pm 12.74$ & $<0.001$ \\
\hline PT $(s, \bar{x} \pm s)$ & $14.61(12.60,16.10)$ & $16.88 \pm 2.90$ & $13.29=1.36$ & $<0.001$ \\
\hline $\mathrm{Cr}(\mu \mathrm{mol} / \mathrm{L}, \bar{x} \pm s)$ & $\begin{array}{l}133.13(98.00 \\
175.00)\end{array}$ & $173.03 \pm 46.41$ & $96.27 \pm 17.13$ & $<0.001$ \\
\hline WBC & $5.48 \pm 3.08$ & $6.95 \pm 3.37$ & $3.89 \pm 1.61$ & $<0.001$ \\
\hline $\mathrm{N} \%$ & $65.52 \pm 16.79$ & $79.61=6.79$ & $50.24 \pm 9.18$ & 0.019 \\
\hline $\operatorname{PLT}(\mathrm{mmol} / \mathrm{L}, \bar{x} \pm \mathrm{s})$ & $\begin{array}{l}83.02<42.00 . \\
109.00\end{array}$ & $73.34 \pm 44.42$ & $93.53 \pm 60.62$ & $<0.001$ \\
\hline Child-Pugh Class A(\%) & $25.95(41 / 158)$ & $10.84(9 / 83)$ & $47.46(32 / 75)$ & $<0.001$ \\
\hline $\mathrm{B}(\%)$ & $40.51(64 / 158)$ & $39.76(33 / 83)$ & $40.68(31 / 75)$ & 0.872 \\
\hline $\mathrm{C}(\%)$ & $33.54(53 / 158)$ & $49.40(41 / 83)$ & $11.86(12 / 75)$ & $<0.001$ \\
\hline Child-Pugh score & $8.93 \pm 2.24$ & $9.81 \pm 2.15$ & $7.29 \pm 1.84$ & $<0.001$ \\
\hline 90 -day mortality, n (\%) & $20.25(32 / 158)$ & $37.35(31 / 83)$ & $1.33(1 / 75)$ & $<0.001$ \\
\hline
\end{tabular}

\section{Comparison of tested biomarkers between cirrhotic patients with sepsis according to the severity}

According to the severity of disease, the cirrhotic patients with sepsis were divided into general sepsis group(group $2, n=33$ ), severe sepsis group (group $2, n=28$ ), and septic shock group (group $3, n=22$ ).no-sepsis group (group $1, n=39$ ). As exhibited in Table 1 ,only the levels of serum sTREM-1 showed increasing trend with the severity of the cirrhotic patients ( Group 3 vs Group 4,P $=0.017$ ). Changes of serum CRP a,PCT,D-lactate and I-FABP levels had no significance with the severity of the cirrhotic patients, even though they was significantly higher in the sepsis group than that in non-sepsis group(Table 2). 
Table 2

Comparison of tested biomarkers between cirrhotic patients with sepsis according to the severity $(n=83)$

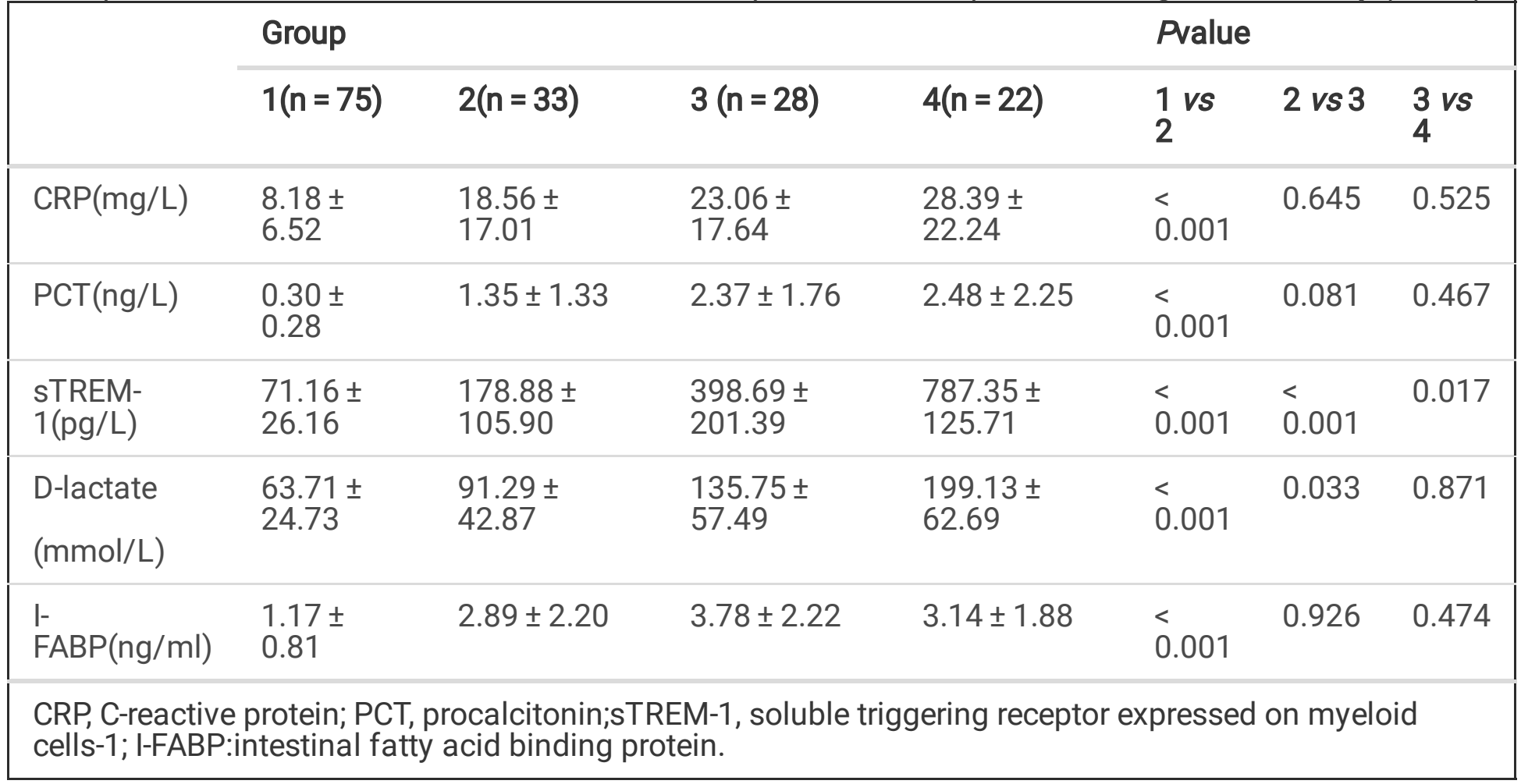

Discriminative power of test biomarkers for the early diagnosis of cirrhotic patients with sepsis upon hospital admission

Figure 1 shows the ROC curves for the CRP, PCT,STREM-1,D-lactateas well as I-FABP, for the early diagnosis of cirrhotic patients with sepsis upon hospital admission. Values for the AUC, cutoff value, sensitivity, specificity, PPV and NPV are listed in Table 3 and Fig. 1. We compared the pairwise AUCs of test biomarkers. sTREM-1 had higher AUCs than CRP $(Z=4.83, P<0.001), \mathrm{PCT}(Z=3.15, P=0.002), \mathrm{D}$-lactateas $(Z=4.06, P<$ $0.001)$ and I-FABP $(Z=4.806, P<0.001)$ (Fig. 1). There was no difference in AUCs between $D$-lactateas and IFABP.(Table 3,Fig. 2) 
Table 3

Diagnostic capability of tested biomarkers as a means of cirrhotic patients with sepsis

\begin{tabular}{|c|c|c|c|c|c|c|c|c|c|}
\hline \multirow[t]{2}{*}{ Biomarkers } & \multirow[t]{2}{*}{ AUC } & \multirow[t]{2}{*}{$\begin{array}{l}\text { Cut- } \\
\text { off }\end{array}$} & \multirow[t]{2}{*}{$\begin{array}{l}\text { Std. } \\
\text { Error }\end{array}$} & \multirow[t]{2}{*}{$\begin{array}{l}P \\
\text { value }\end{array}$} & $\begin{array}{l}\text { Asymptotic } \\
95 \% \\
\text { Confidence } \\
\text { Interval }\end{array}$ & \multirow[t]{2}{*}{ sen } & \multirow[t]{2}{*}{ spe } & \multirow[t]{2}{*}{ PPV } & \multirow[t]{2}{*}{ NPV } \\
\hline & & & & & $\begin{array}{l}\text { Lower limit } \\
\text { Upper limit }\end{array}$ & & & & \\
\hline $\mathrm{CRP}((\mathrm{mg} / \mathrm{L})$ & 0.765 & 9.38 & 0.038 & $<.001$ & $\begin{array}{l}0.692 \\
0.839\end{array}$ & 0.795 & 0.600 & 0.771 & 0.653 \\
\hline PCT(ng/L) & 0.856 & 0.78 & 0.030 & $\begin{array}{l}< \\
0.001\end{array}$ & $\begin{array}{l}0.797 \\
0.915\end{array}$ & 0.687 & 0.893 & 0.663 & 0.893 \\
\hline $\begin{array}{l}\text { sTREM- } \\
1(\mathrm{pg} / \mathrm{ml})\end{array}$ & 0.959 & 123.62 & 0.013 & $<.001$ & $\begin{array}{l}0.934 \\
0.985\end{array}$ & 0.843 & 0.920 & 0.855 & 0.947 \\
\hline $\begin{array}{l}\text { D- } \\
\text { lactate }(\mathrm{mmol} / \mathrm{L})\end{array}$ & 0.815 & 102.16 & 0.033 & $<_{0.001}$ & $\begin{array}{l}0.750 \\
0.880\end{array}$ & 0.602 & 0.920 & 0.614 & 0.933 \\
\hline I-FABP(ng/ml) & 0.766 & 2.38 & 0.038 & $<.001$ & $\begin{array}{l}0.693 \\
0.840\end{array}$ & 0.590 & 0.827 & 0.578 & 0.867 \\
\hline
\end{tabular}

AUC, area under receiver ;sen, sensitivity;spe,specificity;PPV,positive predictive value; NPV, negative predictive value; $C R P, C$-reactive protein ;PCT,Procalcitonin; sTREM-1,Soluble Triggering receptor expressed on myeloid cells 1 ;I-FABP,intestinal fatty acid binding protein

AUC,area under receiver operating characteristic curve;CRP,C-reactive protein ;PCT,Procalcitonin; sTREM1,Soluble Triggering receptor expressed on myeloid cells 1 ;-FABP,intestinal fatty acid binding protein

\section{Ability of biomarkers in predicting prognosis of cirrhotic paients with sepsis}

Of the 83 cirrhotic patients, the total 90-day mortality rate was $37.35 \%(31 / 83)$ according to the Sepsis-3 definitions. The 90-day mortality of cirrhotic patients showed increasing trend with the severity of the sepsis .The mortality of cirrhotic patients with septic shock $(72.73 \%, 16 / 22)$ and severe sepsis( $39.29 \%, 11 / 28)$ was significantly higher than those of general sepsis $(12.12 \%, 4 / 33)(X 2=20.78, P<0.001)$. (Fig. 3). Nonsurvivors had higher sTREM-1 level ( $263.99 \pm 213.79$ vs $672.24 \pm 191.44, P=0.031)$. Changes of serum CRP ,

PCT,D-lactate and I-FABP levels had no significance between survivors and non-survivors $(P=0.179, P=0.240$, $P=0.327, P=0.873$ respectively)(Table 4) 
Table 4

Comparison of tested biomarkers between cirrhotic patients with sepsis in survivors and non-survivors $(n=83)$

\begin{tabular}{|llll|}
\hline Biomarker & $\begin{array}{l}\text { survivors } \\
(\mathbf{n}=\mathbf{5 2})\end{array}$ & non-survivors $(\mathbf{n = 3 1 )}$ & Pvalue \\
\hline CRP $(\mathrm{mg} / \mathrm{L})$ & $19.16 \pm 17.69$ & $29.97 \pm 20.21$ & 0.179 \\
\hline PCT $(\mathrm{ng} / \mathrm{L})$ & $1.56 \pm 1.54$ & $2.74 \pm 1.92$ & 0.240 \\
\hline sTREM-1 $(\mathrm{pg} / \mathrm{L})$ & $263.99 \pm 213.79$ & $672.24 \pm 191.44$ & 0.031 \\
\hline D-lactate $(\mathrm{mmol} / \mathrm{L})$ & $119.00 \pm 62.22$ & $161.27 \pm 71.17$ & 0.327 \\
\hline I-FABP $(\mathrm{ng} / \mathrm{ml})$ & $3.34 \pm 2.34$ & $3.05 \pm 1.69$ & 0.873 \\
\hline
\end{tabular}

CRP, C-reactive protein; PCT, procalcitonin;sTREM-1, soluble triggering receptor expressed on myeloid cells-1; I-FABP:intestinal fatty acid binding protein and D-lactate

\section{Predictability of biomarkers for cirrhotic patients with sepsis for 90-day mortality}

CRP,PCT,STREM-1,D-lactate and I-FABP used to predict the 90-day mortality are described in Table 5 . Figure 3 showed the ROC curves for CRP,PCT,STREM-1,D-lactate and I-FABP for predictability of cirrhotic patients with sepsis for 90-day mortality.sTREM-1 had higher AUCs than CRP $(Z=3.20, P=0.001), \operatorname{PCT}(Z=2.97, P=0.003)$ ,D-lactate $(Z=3.85$,

$P<0.001$ ). The AUCs of I-FABP is only 0.482 and had no value of predicting prognosis.(Table 5 ,Fig. 3 )

Table 5

Predictability of biomarkers for cirrhotic paients with sepsis for 90-day mortality $(n=83)$

\begin{tabular}{|c|c|c|c|c|c|c|c|c|}
\hline \multirow[t]{2}{*}{ Biomarkers } & \multirow[t]{2}{*}{ AUC } & \multirow[t]{2}{*}{$\begin{array}{l}\text { Cutto } \\
\text { ff }\end{array}$} & \multirow[t]{2}{*}{$\begin{array}{l}\text { Std. } \\
\text { Error }\end{array}$} & \multirow[t]{2}{*}{$\begin{array}{l}P \\
\text { value }\end{array}$} & $\begin{array}{l}\text { Asymptotic } 95 \% \\
\text { Confidence Interval }\end{array}$ & \multirow[t]{2}{*}{ sen } & \multirow[t]{2}{*}{ spe } & \multirow{2}{*}{$\begin{array}{l}\text { Youden } \\
\text { (max) }\end{array}$} \\
\hline & & & & & $\begin{array}{l}\text { Lower limit Upper } \\
\text { limit }\end{array}$ & & & \\
\hline $\mathrm{CRP}((\mathrm{mg} / \mathrm{L})$ & 0.675 & 24.49 & 0.063 & 0.008 & 0.5520 .798 & 0.581 & 0.769 & 0.350 \\
\hline PCT(ng/L) & 0.702 & 1.15 & 0.059 & 0.002 & 0.5870 .817 & 0.809 & 0.596 & 0.234 \\
\hline $\begin{array}{l}\text { sTREM- } \\
1(\mathrm{pg} / \mathrm{ml})\end{array}$ & 0.904 & 404.87 & 0.034 & $\begin{array}{l}< \\
0.001\end{array}$ & 0.8360 .971 & 0.903 & 0.827 & 0.730 \\
\hline $\begin{array}{l}\text { D- } \\
\text { lactatemmol/L) }\end{array}$ & 0.625 & 198.44 & 0.064 & 0.058 & 0.5000 .750 & 0.387 & 0.827 & 0.214 \\
\hline I-FABP(ng/ml) & 0.482 & 2.62 & 0.063 & 0.788 & 0.3580 .607 & 0.548 & 0.519 & 0.067 \\
\hline
\end{tabular}

AUC, area under receiver ;sen ,sensitivity;spe,specificity;CRP,C-reactive protein ;PCT,Procalcitonin; sTREM1,Soluble Triggering receptor expressed on myeloid cells $1 ;$;-FABP,intestinal fatty acid binding protein 


\section{Discussion}

Sepsis is a clinical process in which bacterial infection triggers excessive inflammation,and weakened fibers in the immune system.From mild to severe, sepsis can be divided into three stages:sepsis,severe sepsis and septic shock.[24]Infection and sepsis can occur in patients with liver cirrhosis ofvarious etiology(Table 1).The incidence of sepsis significantly promoted the progression of decompensated cirrhosis patients to Acute-on chronic Liver failure(ACLF) $[3,25]$.Organ failure caused by sepsis and sepsis related death are greatly increased in patients with cirrhosis[26]. As we found significant differences in levels of ALB, TB,PT,Cr, PLT count,Child-Pugh class $C$ ratio, Child score, and 90 -daymortality between patients with and without sepsisロTable 1).Cirrhotic patients with sepsiswere in critical conditionand with a poor in-hospital outcome, especially in patientswith Child-Pugh class $C$ and their lower immunity may be responsible for the high incidence of sepsis[27,28 ].The 90-day mortality rate was as high as $37.35 \%(31 / 83)$ in cirrhotic patients with sepsis group.(Table 1). Even if the total number of WBCs and proportion of NEs in sepsis group was higher than that in non-sepsis group,WBC count in two group were within normal range, which may be related to the presence of hyperslenism and decompensated liver cirrhosis.Partial cirrhotic patients haven't any symptoms at the initial stage of infection.It is not easy to attract the attention of clinicians.Some new biomarkers of sepsis would be particularly useful for the early diagnosis in this population.

Unfortunately, early recognition and prognosis prediction of sepsis in patients with cirrhosis is difficult. Serum levels of CRP and PCT have obtained special interest in identifying diagnosis and prediction of bacterial infection in liver cirrhosis[29, 30]. Procalcitonin(PCT) is a sensitive and specific serologic marker, and its level increases at the early stage of liver cirrhosiswith serious bacterial infection, and so has value for early diagnosis [31]. CRP is an acute phase I protein synthesized by hepatocytes when the body is stimulated by microbial invasion or tissue damage. PCT is released by the solid tissue cells of liver and peripheral blood mononuclear cellsduring microbial infection.But Liver failure may lead to the formation ofa network of monocytes to reduce theconcentration of acute-phase proteins (APPs) [32]. The more severe the underlying liver dysfunction, the lower the CRP response to bacteremia [33].JJanos Szederjesi et al reported there was no significant correlation between the levels of the PCT and CRP biomarkers and severe sepsis .Quantitive procalcitonin and C-reactive protein analysis was not shown to be useful in diagnosing severe sepsis[34].Our data showed that levels of CRP and PCT in liver cirrhosis patients with sepsis did not increase significantly (Table 2), even though they were significantly different between the non-sepsis group and sepsis group. levels of CRP and PCT were no meaningful between the non-survival group and in the survival group.

TREM-1 expression can be induced by bacterial products such as lipopolysaccharides and lipoteichoic acid.TREM-1 is expressed on neutrophils and monocytes. It is implicated in the development and amplification of the early inflammatory response to infection and injury. STREM- 1 is the soluble form of this receptor and is released into body fluids when TREM-1 expression is upregulated [35]. As reported by Jedynak and colleagues, the serum level of sTREM-1 measured within the first $24 \mathrm{~h}$ of treatment in the intensive care unit is a useful prognostic biomarker for patients with sepsis, severe sepsis or septic shock [36]. Various studies have suggested that the sTREM-1 concentration in different biologic fluids is significantly higher in patients with bacterial infection than in those with a non-microbial inflammatory process [37-38].sTREM-1 isa reliable biomarker evaluated for the diagnosis and prognosis of sepsis to date 
$[39,40]$. Furthermore, the serum level of sTREM- 1 reflects the severity of sepsis more accurately than that of $C R P, P C T$,is more sensitive for dynamic evaluation of the sepsis prognosis[40, 41]. The serum concentration of sTREM-1 can early predict the 28 -day sepsis mortality $[42,43]$.According to our findings, sTREM-1 was able to predict the development of cirrhotic patients with sepsis. We revealed that cirrhotic patients with sepsis had a significantly increasedserum level of sTREM-1, the highest AUC (0.959), sensitivity of 0.843 , and specificity of 0.920 at a cutoff value of $123.620 \mathrm{ng} / \mathrm{ml}$. Serum levels of sTREM- 1 could be used to predict the early diagnosis of cirrhotic patients with sepsis compared with the levels of CRP, PCT,D-lactate and IFABP(Table 3, Fig. 1).The AUC for predicting the 90-day mortality was 0.904 for the sTREM-1 level,and sTREM-1 levelwas superior to PCT ,CRP,D-lactate and I-FABP in predicting90-day mortality in cirrhotic patients with sepsis.

D-lactate is produced by some bacteria including Klabsilla, Escherichia coli,Lactobacilus species and Bacteroides species. Normally,serum level of D-lactate in mammals are quite low.Serum D-lactate concentration would increase critically ill patients with proven intestinal ischemia[44].D-lactate is a sensitive marker for early diagnosis of gut failure and endotoxemia in patients with cirrhosis.During the event that an ischemia/reperfusion insults, the mucosa is injured and intestinal permeability is increased,leading to an efflux of bacteria and the products of metabolism,including D-lactate into the circulation. The bacterial proliferation would be expected to cause an increased bacterial matabolism with increased production of Dlactate.Serum levels of I-FABP can be used to evaluate intestinal injury and is specifically released by ischemic mucosa. In the past decades studied reported that D-lactate and I-FABP may the best candidate markers for early diagnosis of acute mesenteric ischemia[45]. Our study showed levels of D-lactate and IFABP were meaningful in the sepsis group than in the non-sepsis group.However there was no significant difference between patients with severe sepsis and septic shock,the same result was found in survivors group and non-survivors group.lt was obvioused sepsis could further increased mucosal intestinal permeability in patients with cirrhosis,but the level of serum D-lactate and I-FABP was not related to the severity of sepsis and couldn't predict 90-day mortality in cirrhotic patients with sepsis.

Our study showed thatthe serum level of sTREM-1 wasa suitable biomarker for identification of cirrhotic patientswith septic. Moreover, the serum level ofsTREM-1 may be an outcome predictor in cirrhotic patientswith sepsis, and could act as an indicator for starting early therapeutic interventions. Recently some data demonstrate that the TREM-1 pathway on Kupffer cells plays an essential role in hepatic inflammation and fibrogenesis in a mouse model of fibrosis. TREM-1 controls the mobilization of inflammatory cells in response to injury and consequently enhances liver damage[46]. Maybe we will study the relationship between TREM-1 and severity of liver inflammation in the future.

Our study had two main limitations. First, in this study ,only serum biomarker levels were detected within 12 hours of hospitalization and there is a lack of continuous dynamic change trend.Second,our study cohort was relatively small. Further validation of a larger dataset is required. Third, cirrhotic patients with sepsis' condition was complex,and the intervention measures before hospitalization were different.Some critically ill patients with severe liver function damage may have metabolic disorders and affect the results.

\section{Conclusions}


The CRP,PCT, sTREM-1, D-lactate and I - FABP were helpful for the early diagnosis in liver cirrhotic patients with sepsis.Serum STREM-1 is a valuable new biomarker for early diagnosis and prognosis in cirrhosis with sepsis, proving more accurate than CRP,PCT, D - lactate and I - FABP.Serum D - lactate and I - FABP levels can identify early cirrhosis withsepsis, but they is little relationship with the prognosis of patients.

\section{Abbreviations}

HBV, Hepatitis B virus

HCV, Hepatitis C virus

HEV Hepatitis E virus

ALT, alanine aminotransferase;

ALB, albumin

$\mathrm{TB}$, total bilirubin

PT, prothrombin time

$\mathrm{Cr}$, creatinine

WBC, white blood count

$\mathrm{N} \%$, proportion of neutrophils

PLT, platelet

AUC,area under receiver operating characteristic curve

CRP,C-reactive protein

PCT,Procalcitonin

sTREM-1,Soluble Triggering receptor expressed on myeloid cells 1

I-FABP,intestinal fatty acid binding protein

\section{Declarations}

\section{Ethics approval and consent to participate}

The study protocol was established, according to the ethical guidelines of the

Helsinki Declaration and was approved by the Human Ethics Committee of

Zhejiang Provincial People's Hospital. Written informed consent was obtained 
from individual or guardian participants.

\section{Consent for publication}

Not applicable

\section{Availability of data and materials}

All data generated or analysed during this study are included in this published article.

\section{Conflicts of interests}

The authors have declared that no competing interests exist.

\section{Funding}

This work was supported byNatural Science Foundation of Zhejiang

province(LY18H190002) and project of science and technology of traditional

Chinese Medicine in Zhejiang Province『2016ZA026).

Obtained funding:Danhong Yang

\section{Author Contributions}

Conception and design of experiments: Danhong Yang and Hongying Pan.

Analyses of data: Danhong Yang and Qiang Zhang. Carried out the experiments:

Qiang Zhang, Weiping Wang, Yicheng Huang and Miaomiao Wu. Wrote

the manuscript:Danhong Yang.

\section{Acknowledgements}

We appreciated Yasheng He for data statistics the manuscript.

\section{References}

1. Foreman MG,Mannino DM,Moss M. Cirrhosis as a risk factor for sepsis and death: analysis of the national hospital discharge survey. Chest. 2003; 124『3®:1016- doi: 10.1378/chest.124.3.1016

2. BrunsT, Zimmermann HW, Stallmach A. Risk factors and outcome of bacterial infections in cirrhosis. World J Gastroenterol. 2014; 20ه10邓:2542- doi: 10.3748/wjg.v20.i10.2542 
3. Shi Y,Yan H,Zhou Z. Clinical course of cirrhosis patients hospitalized for acute hepatic deterioration: a prospective bicentric study. J Med (Baltimore). 2015; 94: e2031. doi: 10.1097/MD.0000000000002031

4. Angeli P,Tonon M, Pilutti C. Sepsis induced acute kidney injury in patients with cirrhosis. Hepatol Int. 2016; 10(1): 115- doi: 10.1007/s12072-015-9641-1

5. Yang D, XieY, Pan H. Clinical characteristics and prognostic factors of liver cirrhosis patients with systemic inflammatory response syndrome. Hepatology Research. 2017 ;47(11):1174-85. https://doi.org/10.1111/hepr.12886

6. Slivestre JP,Coelho LM,Povoa PM.Impact of fulminant hepatic failure in C-reactive protein? Crit Care. 2010;25(4):657e7-12. doi:10.1016/j.jcrc.2010.02.004

7. Bouchon A, Dietrich J, Colonna M. Cutting edge: inflammatory responses can betriggered by TREM-1, a novel receptor expressed on neutrophils and monocytes. Immunol. 2000; 164ه10®:4991-

doi: 10.4049/jimmunol.164.10.4991

8. Routsi C, Giamarellos-Bourboulis EJ, Antonopoulou A, Kollias S, Siasiakou S,Koronaios A, Zakynthinos S, Armaganidis A,et al.

Does soluble triggering receptor expressed on myeloid cells-1 play anyrole in the pathogenesis of septic shock? Clin Exp Immunol. 2005; 142:62-7.

doi: 10.1111/j.1365-2249.2005.02887.x

9. Pelham CJ,Pandya AN,Agrawal DK.Triggering receptor expressed on myeloid cells receptor family modulators:a patient review.Expert Opin Ther Pat.2014;24(12):1383-95. doi: 10.1007/s11883-009-00364

10. Barati M, Bashar FR, Shahrami R.Soluble triggering receptor expressed on myeloid cells 1 and the diagnosis of sepsis. Crit Care. 2010; 25囚2囚: 362e1 -6. DOI: 10.1016/j.jcrc.2009.10.004

11. Zhang J, She D, Feng D. Dynamic changes of serum soluble triggering receptor expressed on myeloid cells-1 (sTREM-1) reflect sepsis severity and can predictprognosis:a prospective study. BMC Infect Dis.2011;11: 53.

doi: $10.1186 / 1471-2334-11-53$

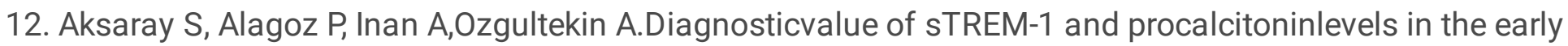
diagnosis of sepsis.North Clin Istanb. 2017 ;3(3):175-82. doi: 10.14744/nci.2016.26023

13. Larsen FF, Petersen JA.Novel biomarkers for sepsis: A narrative review.

Eur J Intern Med. 2017 ;45:46-50. DOI: 10.1016/j.ejim.2017.09.030

14. Jedynak M, SiemiatkowskiA, MroczkoB, Groblewska M, Milewski R,SzmitkowskiM.SolubleTREM1 Serum Level can Early PredictMortality of Patients with Sepsis, Severe Sepsis and Septic Shock.Arch Immunol Ther Exp (Warsz). 2018 ;66(4):299-306. doi: 10.1007/s00005-017-0499-x

15. Aguirre Valadez JM, Rivera-Espinosa L, Méndez-Guerrero O, Chávez-Pacheco JL, García-Juárezl. TorreA .Intestinal permeability in a patient with liver cirrhosis.Ther Clin Risk Manag.2016;12:1729-48. 
16. Nicoletti A, Ponziani FR, Biolato M,Valenza V, Marrone G, Sganga G, et al.Intestinal permeability in the pathogenesis of liver damage:From non-alcoholic fatty liver disease to liver translantation.World $\mathrm{J}$ Gastroenterol .2019.2533(33):4814-34. doi: 10.3748/wjg.v25.i33.4814

17. Nielsen C,Mortensen FV,Erlandsen EJ『L-and D-lactate

as biomarkers of arterial-induced intestinal ischemia囚an experimental study in pigs.Int J Surg.2012;10(6)凹 296-300. doi: 10.1016/j.ijsu.2012.05.003

18. Van der Voort PH, Westra B, Wester JP, Bosman RJ, van Stijn I, Haagen IA, Loupatty FJ, Rijkenberg S.Can serum L-lactate, D-lactate, creatine kinase and I-FABP be used as diagnostic markers in critically ill patients suspected for bowel ischemia.BMC Anesthesiol. 2014;14:111.

doi: $10.1186 / 1471-2253-14-111$

19. Güzel M, Sözüer EM, Salt Ö, İkizceli İ, Akdur O, Yazici C.Value of the serum I-FABP level fordiagnosing acute mesenteric ischemia. 2014;44(11):2072-6. doi: 10.1007/s00595-013-0810-3

20. MontagnanaM, Danese E, Lippi G.Biochemical markers of acute intestinal ischemia:possibilities and limitations.Ann Transl Med. $2018 ; 6(17): 341$.

doi: $10.21037 /$ atm.2018.07.22

21. Obulkasim Memet, Lin Zhang, Jie Shen.Serological biomarkers for acute mesenteric ischemia.Ann Transl Med. $2019 ; 7(16): 394$.

doi: $10.21037 /$ atm.2019.07.51

22. Singer M, Deutschman CS, Seymour CW, Shankar-Hari M, Annane D, Bauer M,et al.The Third International Consensus Definitions for Sepsis and Septic Shock(Sepsis-3). 2016 ;315(8):801-10.

doi: $10.1001 /$ jama.2016.0287

23. Shankar-Hari M, Phillips GS, Levy ML, Seymour CW, Liu VX, Deutschman CS, et al.Developing a New Definition and Assessing New Clinical Criteria for SepticShock:For theThird International Consensus Definitions for Sepsis and Septic Shock (Sepsis-3). 2016;23;315(8):775-87.

doi: 10.1001/jama.2016.0289

24. Pavel Strnad, Frank Tacke, Alexander Koch, Christian Trautwein. Liver - guardian, modifier and target of sepsis.Nat Rev Gastroenterol Hepatol. 2017 ;14(1):55-66. doi: 10.1038/nrgastro.2016.168

25. Salvatore Piano, Alessandra Brocca, Sara Mareso, Paolo Angeli.Infections complicating cirrhosis .Liver Int. $2018 ; 38$ ( Suppl 1):126-33.

doi: 10.1016/j.cgh.2019.09.035 
26. Douglas A Simonetto, Laura Piccolo Serafim, Alice Gallo de Moraes, Ognjen Gajic, Patrick S Kamath.Management of Sepsis in Patients With Cirrhosis: Current Evidence and Practical Approach.Hepatology. 2019;70(1):418-28.

doi: 10.1002/hep.30412

27. Rafael Oliveira Ximenes, AlbertoQueiroz Farias, Augusto Scalabrini Neto, Márcio Augusto Diniz,et al.Patients with cirrhosis in the ED: early predictors of infection and mortality.Am J Emerg Med.2016;34(1):25-9.

doi: 10.1016/j.ajem.2015.09.004

28. Gary Aguilar Saldaña. Predictors of infection and mortality in patients with liver cirrhosis in the high complexity hospital. Rev Gastroenterol Peru. 2019;39(1):55-63. PMID: 31042237.

29. Bota DP, Van Nuffelen M, Zakariah AN. Serum levels of C-reactive protein and procalcitonin in critically ill patients with cirrhosis of the liver.Lab Clin Med. 2005; 146:347-51. doi: 10.1016/j.lab.2005.08.005

30. Lazzarotto C, Ronsoni MF, Fayad L.Acute phase proteins for the diagnosis of bacterial infections and prediction of mortality in acute complications of cirrhosis. Ann Hepatol. 2013; 1(4): 599-607.

https://doi.org/10.1016/S1665-2681(19)31344-4

31. Cai ZH, Fan CL, Zheng JF.Measurement of serum procalcitonin levels for the

early diagnosis of spontaneous bacterial peritonitis in patients withdecompensated liver cirrhosis. BMC Infect Dis. 2015;13;(15):55.

doi: $10.1186 / s 12879-015-0776-4$

32. Papp M, Vitalis Z, Altorjay I. Acute phase proteins in the diagnosis and prediction of cirrhosis associated bacterial infections. Liver Int. 2012; 32: 603-11. doi: 10.1111/j.1478-3231.2011.02689.x

33. Park WB, Lee KD, Lee CS. Production of C-reactive protein in Escherichia coliinfected patients with liver dysfunction due to liver cirrhosis. Diagn Microbiol Infect Dis. 2005; 51: 227-30.

doi: 10.1016/j.diagmicrobio.2004.11.014

34. JJanos Szederjesi, Emoke Almasy 1, Alexandra Lazar, Adina Huțanu, ludita Badea, Anca Georgescu.An Evaluation of Serum Procalcitonin and C-Reactive Protein Levels as Diagnostic and Prognostic Biomarkers of Severe Sepsis.Crit Care Med (Targu Mures). 2015;1(4):147-53.

doi: $10.1515 / \mathrm{jccm}-2015-0022$

35. Gibot S, Cravoisy A.Soluble form of the triggering receptor expressed on myeloid cells-1 as a marker of microbial infection.Clin Med Res. 2004 ;2(3):181-7. doi: 10.3121/cmr.2.3.181

36. Jedynak M,Siemiatkowski A,Mroczko B.Soluble TREM-1 Serum Level can EarlyPredict Mortality of Patients with Sepsis, Severe Sepsis and Septic Shock.Arch Immunol Ther Exp (Warsz).2017; 27:1-8. 
37. Plachouras D, Routsi C, Giamarellos-Bourboulis EJ.Monocytes as a site of production of soluble triggering receptor expressed on myeloid cells-1 (sTREM-1) in the septic host.Scand J Infect Dis. 2006;38(10):909-15.

doi: $10.1080 / 00365540600786523$

38. Liu CL, Hsieh WY, Wu CL.Triggering receptor expressed on myeloid cells-1 in pleural effusions: a marker of inflammatory disease.Respir Med. 2007;101(5):903-13. doi: 10.1016/j.rmed.2006.09.021

39. Larsen FF, Petersen JA. Novel biomarkers for sepsis: A narrative review.Eur J Intern Med. 2017;45:46-50. doi: 10.1016/j.ejim.2017.09.030

40. Ríos-Toro JJ, Márquez-Coello M, García-Álvarez JM, Martín-Aspas A, Rivera-Fernández R, Sáez de Benito A, Girón-González J.A Soluble membrane receptors, interleukin 6, procalcitonin and C reactive protein as prognostic markers in patients with severe sepsis and septic shock.PLoS One. 2017;12(4):e0175254. doi: 10.1371/journal.pone.0175254

41. Su L, Han B, Liu C.Value of soluble TREM-1, procalcitonin, and C-creative protein serum levels as biomarkers for detecting bacteremia among sepsis patients with new fever in intensive care units: a prospective cohort study. BMC Infect Dis. 2012; 12(1):157. doi: 10.1186/1471-2334-12-157

42. Jedynak M, Siemiatkowski A, Mroczko B. Soluble TREM-1 Serum Levelcan Early Predict Mortality of Patients with Sepsis, Severe Sepsis and Septic Shock.Arch Immunol Ther Exp (Warsz).2018; 66(4): 299doi: $10.1007 /$ s00005-017-0499-x

43. Van der PH,Westra B,Westra B,Wester JP,Bosman RJ,et al. Canserum L-latate,D-lactate,creatine kinase and I-FABP be used as diagnostic markers in critically ill patients suspected for bowel ischemia? BMC Anesthesiol.2014;14:111. doi:10.1186/1472-2253-14-111

44. Memet O,Zhang L,Shen J.Serological biomarkers for acute mesentericischemia.Ann Transl Med.2019,7(16):394. doi:10.21037/am.2019.07.51

45. Nguyen-Lefebvre AT, Ajith A, Portik-Dobos V.The innate immunereceptorTREM-1 promotes liver injury and fibrosis.J Clin Invest. 2018 ;128(11):4870-83. doi: 10.1172/JCI98156

\section{Figures}




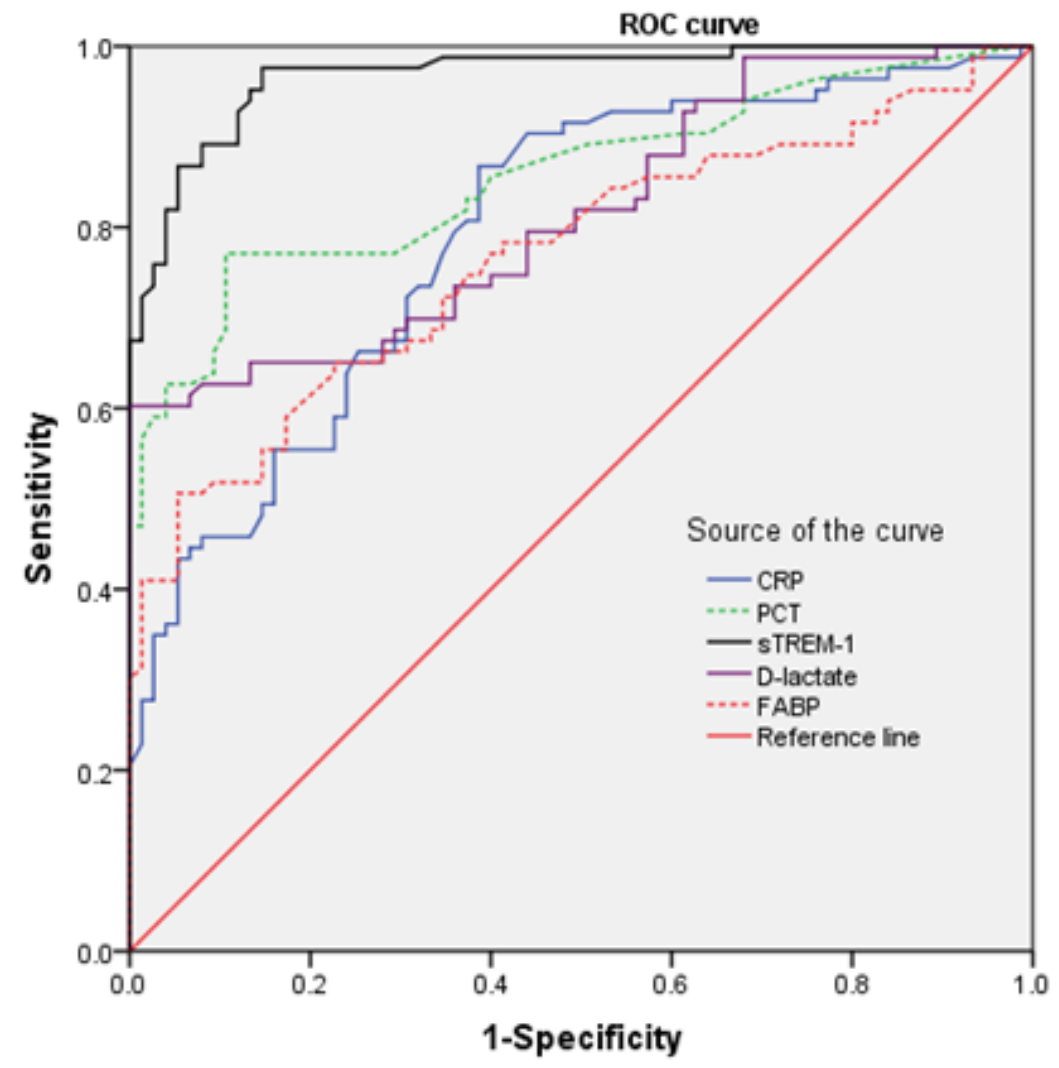

Figure 1

The ROC curves for the test biomarkers on admission for the identification of cirrhotic patients with sepsis.sTREM-1 had the highest AUC and accuracy, followed by PCT .

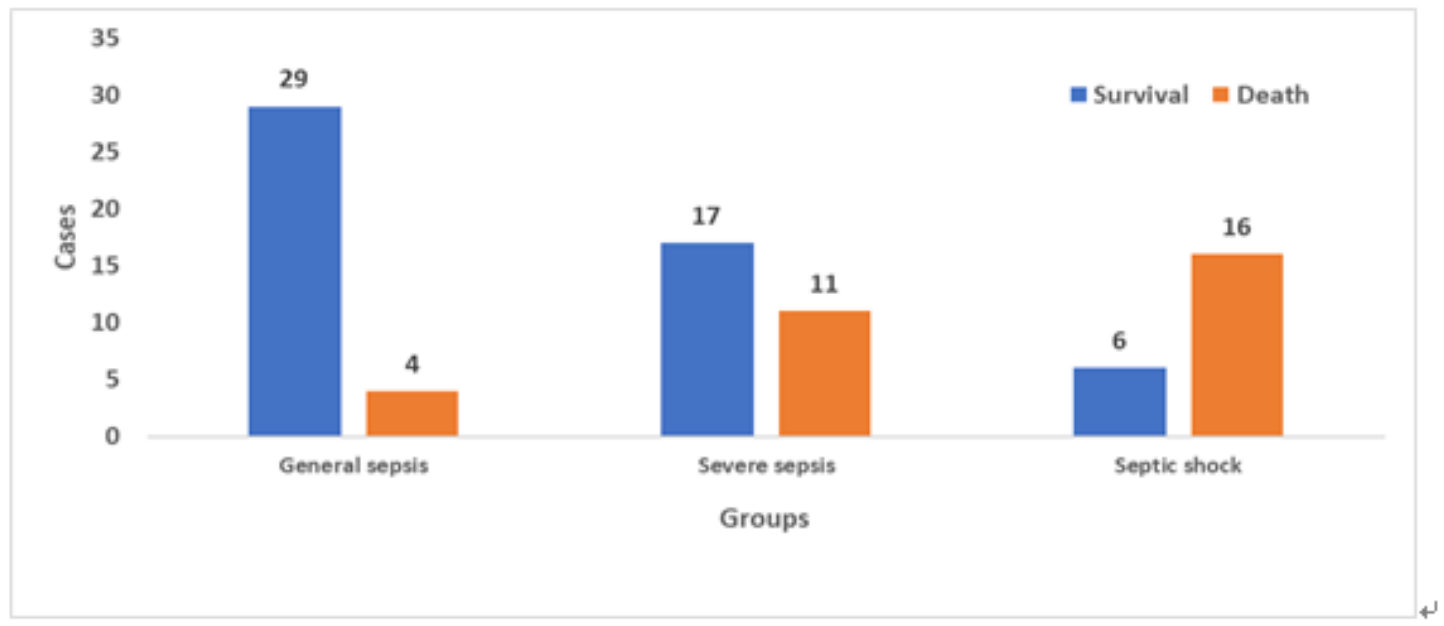

\section{Figure 2}

The 90-day mortality of cirrhotic patients showed increasing trend with the severity of the sepsis 


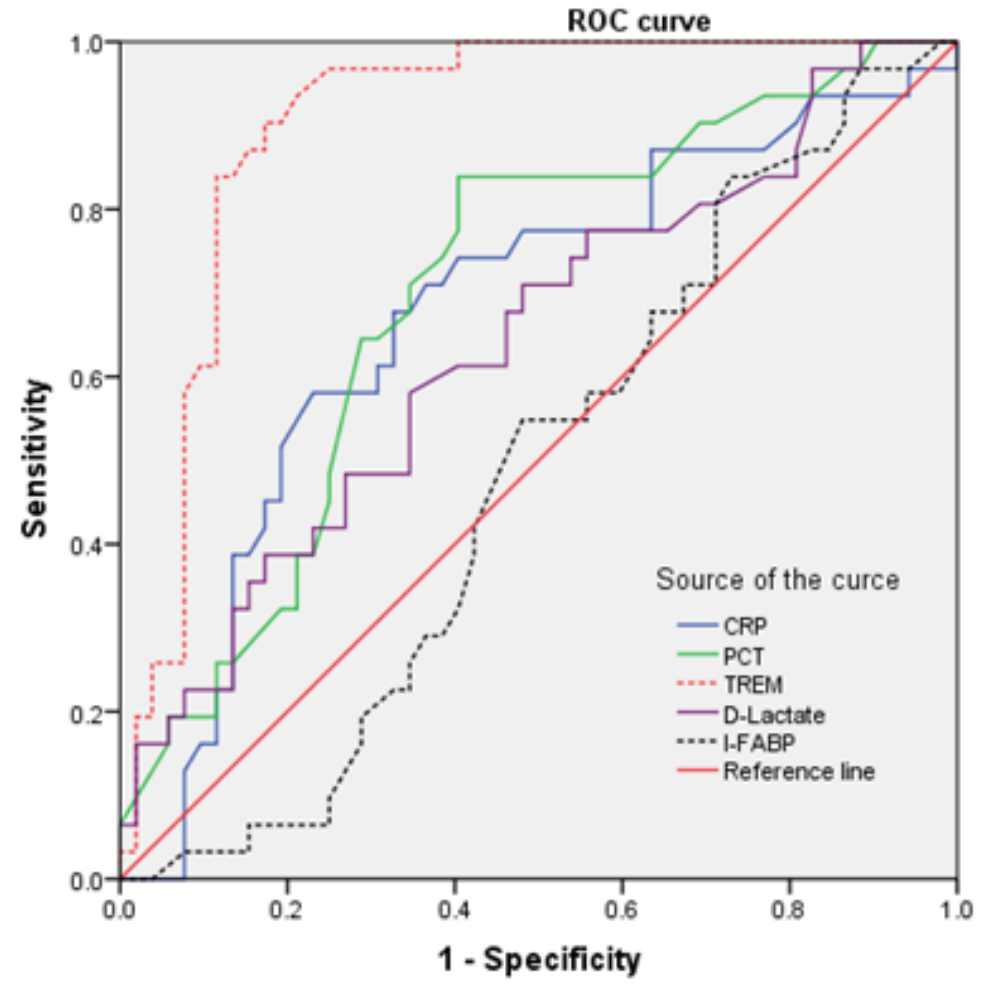

\section{Figure 3}

The ROC curves for the test biomarkers on admission for predictors of 90-day death of cirrhotic patients with sepsis.sTREM-1 had the highest AUC and accuracy, followed by PCT . AUC,area under receiver operating characteristic curve;CRP,C-reactive protein ;PCT,Procalcitonin; sTREM-1,Soluble Triggering receptor expressed on myeloid cells $1 ;$;-FABP,intestinal fatty acid binding protein 